\title{
Ethylene-forming enzyme and bioethylene production
}

\author{
Carrie Eckert ${ }^{1,3}$, Wu Xu², Wei Xiong ${ }^{1}$, Sean Lynch ${ }^{1,3}$, Justin Ungerer ${ }^{1}$, Ling Tao ${ }^{1}$, Ryan Gill ${ }^{3}$, Pin-Ching Maness ${ }^{1 *}$ \\ and Jianping $\mathrm{Yu}^{1 *}$
}

\begin{abstract}
Worldwide, ethylene is the most produced organic compound. It serves as a building block for a wide variety of plastics, textiles, and chemicals, and a process has been developed for its conversion into liquid transportation fuels. Currently, commercial ethylene production involves steam cracking of fossil fuels, and is the highest $\mathrm{CO}_{2}$-emitting process in the chemical industry. Therefore, there is great interest in developing technology for ethylene production from renewable resources including $\mathrm{CO}_{2}$ and biomass. Ethylene is produced naturally by plants and some microbes that live with plants. One of the metabolic pathways used by microbes is via an ethylene-forming enzyme (EFE), which uses a-ketoglutarate and arginine as substrates. EFE is a promising biotechnology target because the expression of a single gene is sufficient for ethylene production in the absence of toxic intermediates. Here we present the first comprehensive review and analysis of EFE, including its discovery, sequence diversity, reaction mechanism, predicted involvement in diverse metabolic modes, heterologous expression, and requirements for harvesting of bioethylene. A number of knowledge gaps and factors that limit ethylene productivity are identified, as well as strategies that could guide future research directions.
\end{abstract}

Keywords: Ethylene-forming enzyme, Bioethylene, Diversity, Mechanism, Heterologous expression

\section{Introduction}

The rising global demand for petroleum, its restricted supply base, and its deleterious effects on the environment has prompted the development of infrastructurecompatible renewable fuels and chemicals. One potentially renewable feedstock that could have an impact is ethylene. In 2011, the global production capacity of ethylene was 142 million metric tonnes and is forecast to reach 165 million metric tonnes, with an economic impact of US\$200 billion per year, by 2015 [1]. Ethylene is the most widely used feedstock in several industries including plastics, textiles, and solvents. In addition, ethylene can also be catalytically polymerized to gasoline-rich hydrocarbons in the $\mathrm{C} 5-\mathrm{C} 10$ range $[2,3]$. Ethylene is currently produced from steam cracking of fossil fuels or from dehydrogenation of ethane, representing the largest $\mathrm{CO}_{2}-$ emitting process in the chemical industry. By current state of the art technology, $2 \mathrm{MJ}$ of energy are invested per

\footnotetext{
*Correspondence: pinching.maness@nrel.gov; jianping.yu@nrel.gov ${ }^{1}$ Biosciences Center, National Renewable Energy Laboratory, 15013 Denver West Parkway, Golden, CO 80401, USA

Full list of author information is available at the end of the article
}

pound of ethylene made; given the ethylene industry's massive size, this product alone accounts for $1.5 \%$ of United States' carbon footprint [4]. A renewable route to ethylene production would therefore fulfill an enormous energy and chemical market while helping to preserve the environment.

Ethylene can also be produced biologically. It is a plant hormone that modulates growth and development, and functions in the defense response to abiotic or biotic stress including pathogen attack $[5,6]$. In plants, ethylene is produced in a two-step reaction from methionine via S-adenosyl-methionine (SAM). SAM is first converted to1-aminocyclopropane-1-carboxylic acid (ACC) by ACC synthase. ACC oxidase then catalyzes the oxidative release of ethylene and cyanide $(\mathrm{CN})$. Although $\mathrm{CN}$ is converted to $\beta$-cyanoalanine to avoid toxicity in plants, utilization of this pathway for biotechnological ethylene production by other organisms is limited by the need for $\mathrm{CN}$ mitigation.

In addition to plants, a variety of microbes including bacteria and fungi also produce ethylene, probably as a causal agent in plant diseases [7]. In Escherichia coli, 
Cryptococcus albidus, and a variety of other bacteria, ethylene is spontaneously produced at trace amounts via oxidation of 2-keto-4-methylthiobutyric acid (KMBA), a transaminated derivative of methionine produced in an NADH:Fe(III)EDTA oxidoreductase-mediated reaction that is enhanced under ammonia limitation $(\mathrm{C} / \mathrm{N}=20)$ $[8,9]$. Formation of KMBA is proposed as a means to recover amino nitrogen from methionine, resulting in the spontaneous production of ethylene from KMBA. A third type of ethylene pathway found in Pseudomonas syringae and Penicillium digitatum utilizes $\alpha$-ketoglutarate (AKG) and arginine as substrates in a reaction catalyzed by an ethylene-forming enzyme (EFE) [10-14], which will be the focus of this review.

Heterologous expression of a single efe gene from $P$. syringae resulted in ethylene production in a number of hosts including E. coli $[15,16]$, Saccharomyces cerevisiae [17], Pseudomonas putida [18], Trichoderma viride [19], Trichoderma reesei [20], tobacco [21], and cyanobacteria [22-26]. These hosts utilize a variety of carbon sources including lignocellulose and $\mathrm{CO}_{2}$, highlighting the various feedstocks that could potentially be used for bioethylene production. In addition, ethylene is not toxic to these organisms, and as a gas it separates easily from cultures. These features compare favorably with other biofuel products such as alcohols or lipids, which tend to be toxic and/or are costly to separate. However, further fundamental and applied studies are needed to bring bioethylene technology to commercial scales. Emerging research topics include a more in-depth understanding of EFE structure and reaction mechanisms, metabolic engineering strategies to improve productivity, and ethylene harvesting technologies. This review aims to provide a summary of the existing literature and to present our own analysis of enzymes and pathways, which together outline a strategy for future research and development of bioethylene production.

\section{EFE discovery}

Ethylene is a hormone that regulates multiple aspects of growth and stress response in plants, and is also a common metabolic product of many fungi and bacteria that live with plants. The common green mold on citrus fruits, $P$. digitatum, was one of the first identified ethylene-producing microbes [10-13]. A cell-free system was prepared from $P$. digitatum [27], and EFE was subsequently purified as a $42-\mathrm{kD}$ protein that required ferrous iron, oxygen, AKG, and arginine for ethylene production [28]. This reaction is in contrast to that in higher plants, which utilize methionine as a precursor in a two-enzyme reaction.

Bacterial production of ethylene was first reported in Pseudomonas solanacearum strains, which are involved in early ripening of banana fruits or in wilting of tobacco and tomato [29]. The most efficient microbial ethylene producers include certain pathovars of $P$. syringae. Two of the most studied strains include $P$. syringae pv. phaseolicola PK2 (Kudzu strain) and P. syringae pv. glycinea, which cause halo blight of the vine weed Kudzu and soybean, respectively [7]. Using a cell-free system prepared from the Kudzu strain, it was determined that a 42-kD EFE monomer was required for a reaction utilizing ferrous iron, oxygen, AKG, and arginine, in agreement with EFE studies in the mold $P$. digitatum [14]. Despite the variance of the $P$. digitatum and Kudzu N-terminal sequences [14], the two proteins share overall sequence similarity (see below). The Kudzu efe gene was localized to an indigenous plasmid, and when this gene was cloned and expressed in E. coli, ethylene production was detected, verifying that expression of a single gene is sufficient for ethylene production in heterologous hosts [15].

\section{EFE sequence diversity and features}

To date, sequence diversity of EFEs has not been reviewed, despite the enormous body of available sequencing data. We therefore constructed a phylogenetic tree of EFE based on sequences from the NCBI database with more than $40 \%$ identity to that of Kudzu (Figure 1). These sequences can be divided into two major groups and a minor group, with pairwise sequence alignments revealing approximately $25 \%$ identity and $65 \%$ similarity overall, with the central regions exhibiting the highest conservation ( $\mathrm{Wu} \mathrm{Xu}$, unpublished data). The identified sequences were annotated as ethylene (succinate)-forming enzyme, 2-oxoglutarate (2OG)-Fe(II) oxygenase, hypothetical protein, 1-aminocyclopropane-1-carboxylic acide oxidase (ACCO), ACC deaminase, and oxidoreductase, suggesting that more functional studies are needed to accurately classify the EFE and EFE-related sequences. As EFEs and ACCOs both catalyze an ethylene formation reaction and belong to the superfamily of 2OG/Fe(II)dependent hydroxylase enzymes [30], they may share common structural features. To identify conserved amino acids, representative ACCO sequences from the Protein Data Bank (PDB) were compared with Kudzu EFE. The top two enzymes identified are an ACCO from petunia, the only ACCO whose structure has been experimentally determined (PDB ID: 1W9Y; 29.7\% similarity and 18.1\% identity) [31], and a putative 2OG-Fe(II) oxygenase from Caulobacter crescentus (PDB ID: 3OOX; 26.1\% similarity and $17.1 \%$ identity). ACCOs from Arabidopsis thaliana and Zea mays, and 2OG-Fe(II) oxygenases from the cyanobacteria Anabaena variabilis and Nostoc punctiforme were also selected for comparison (Figure 2). The cyanobacterial sequences are of particular interest because of the possibility that their 2OG-Fe(II) oxygenases function as EFE; these cyanobacteria are symbiotic nitrogen-fixing 


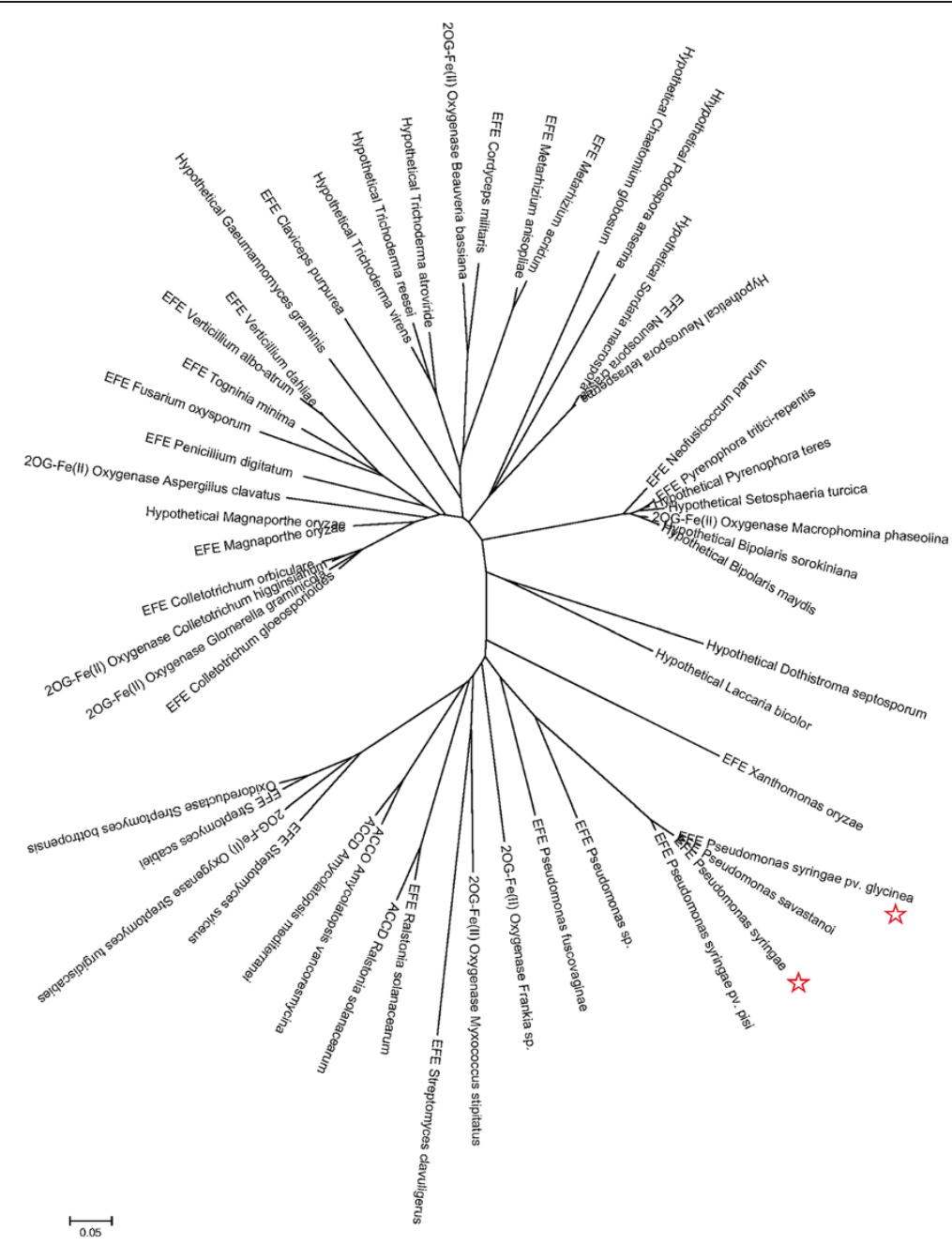

Figure 1 Phylogenetic study of ethylene-forming enzyme (EFE) and EFE-homologous sequences using MEGA 5.2. The tree could be divided into two large groups and one small group. Pseudomonas syringae is the Kudzu strain. EFEs that are heterologously expressed are marked with red stars.

partners with plants, and ethylene production could facilitate the establishment of symbiosis. We found 17 amino acids conserved between Kudzu EFE, ACCOs, and 2OG-Fe(II) oxygenases (Figure 2, highlighted in yellow), suggesting that these residues may play important roles in enzyme structure/function. It has been reported that a majority of the active sites of 2OG/Fe(II)-dependent hydroxylase enzymes contain a single ferrous ion bound in a tridentate ligand arrangement, which is referred to as "a triad of His-Asp/Glu-His." In the petunia ACCO, His177, Asp179, and His234 form this triad [31]. Based on our sequence alignment, a putative ferrous ion binding site of the Kudzu EFE may consist of three conserved amino acids out of the total of seventeen identified: His189, Asp191, and His268. These three amino acids are physically close in our putative 3D EFE model (Wu Xu, unpublished data). The Kudzu EFE contains 10 His residues, and when site-directed mutagenesis of His to Gln of each was carried out, this resulted in 0 to $60 \%$ of activity compared with wild type [32]. Interestingly, mutation of His268 and His189, identified here as possible triad residues, showed enzyme activity of $0 \%$ and $1.8 \%$, respectively. Thus, H189, D191, and H268 are the most likely residues for the triad moiety of EFE in the Kudzu strain. Further studies are needed to test this hypothesis.

\section{EFE reaction mechanism and stoichiometry}

Studies of the EFE reaction using cell-free extracts of the fungus $P$. digitatum [27] and the Kudzu strain [33] have led to the following equation for EFE-dependent ethylene production:

\section{AKG $+3 \mathrm{O}_{2}+\mathrm{L}$-arginine $\rightarrow 2$ ethylene + succinate +7 $\mathrm{CO}_{2}+$ guanidine + $\mathrm{P} 5 \mathrm{C}$}

Based on the substrate ratio of 3:1 for AKG and arginine and the product ratio of 2:1:1 for ethylene, succinate, and 


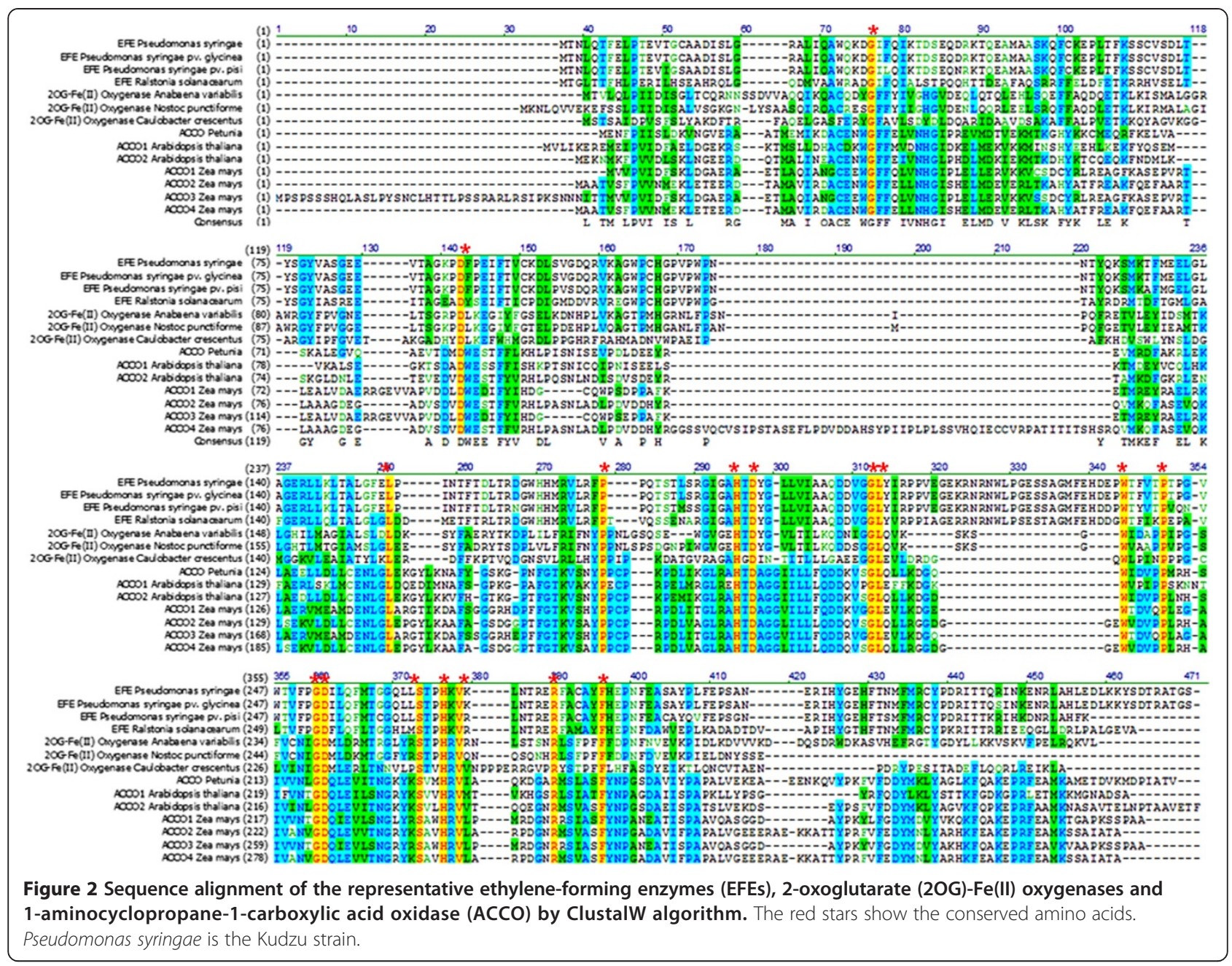

L-delta 1-pyrroline-5-carboxylate (P5C), Fukuda et al. proposed a unique dual-circuit mechanism in which EFE catalyzes two different reactions in a 2:1 ratio [33]. In the first (main) reaction (two cycles), arginine remains bound as a cofactor while two AKG are converted to six $\mathrm{CO}_{2}$ and two ethylene. In the second (sub-)reaction (one cycle), both AKG and arginine are consumed to yield P5C, guanidine, succinate, and $\mathrm{CO}_{2}$. Despite accounting for all of the components added/detected in these in vitro studies, the proposed reaction scheme only partially fits the mechanism determined for other related and wellstudied enzymes in the superfamily of $2 \mathrm{OG} / \mathrm{Fe}(\mathrm{II})-$ dependent hydroxylases. The reactions of the latter involve the oxidative decomposition of AKG to $\mathrm{CO}_{2}$ and succinate while coupling to the hydroxylation of a co-substrate such as arginine to hydroxyarginine [30]. To address inconsistencies in the EFE reaction mechanism, studies utilizing modern analytical techniques are needed. If the dual-circuit mechanism is correct, is the ethylene-producing catalytic cycle necessarily coupled to the succinate-producing catalytic cycle, or is EFE a promiscuous enzyme capable of catalyzing two distinct reactions, with one being the hydroxylation of arginine and the second the degradation of AKG into $\mathrm{CO}_{2}$ and ethylene? If the two reactions are separable, then it may be possible to engineer EFE to produce ethylene without the wasteful generation of side products.

\section{EFE reaction and cellular carbon flux}

Although EFE reaction stoichiometry and the proposed dual-circuit mechanism need further verification, they provide a starting point to analyze the EFE reaction within metabolic networks, providing a systematic overview to enhance understanding and guide engineering approaches.

Efficient ethylene biosynthesis should involve metabolic pathways for which the molar yield (product:substrate molar ratio) is maximal for ethylene and minimal for byproducts and energy/cofactor consumption. Elementary node analysis [34,35] was applied in this study, and Figure 3 shows theoretical yields and cofactor costs for 


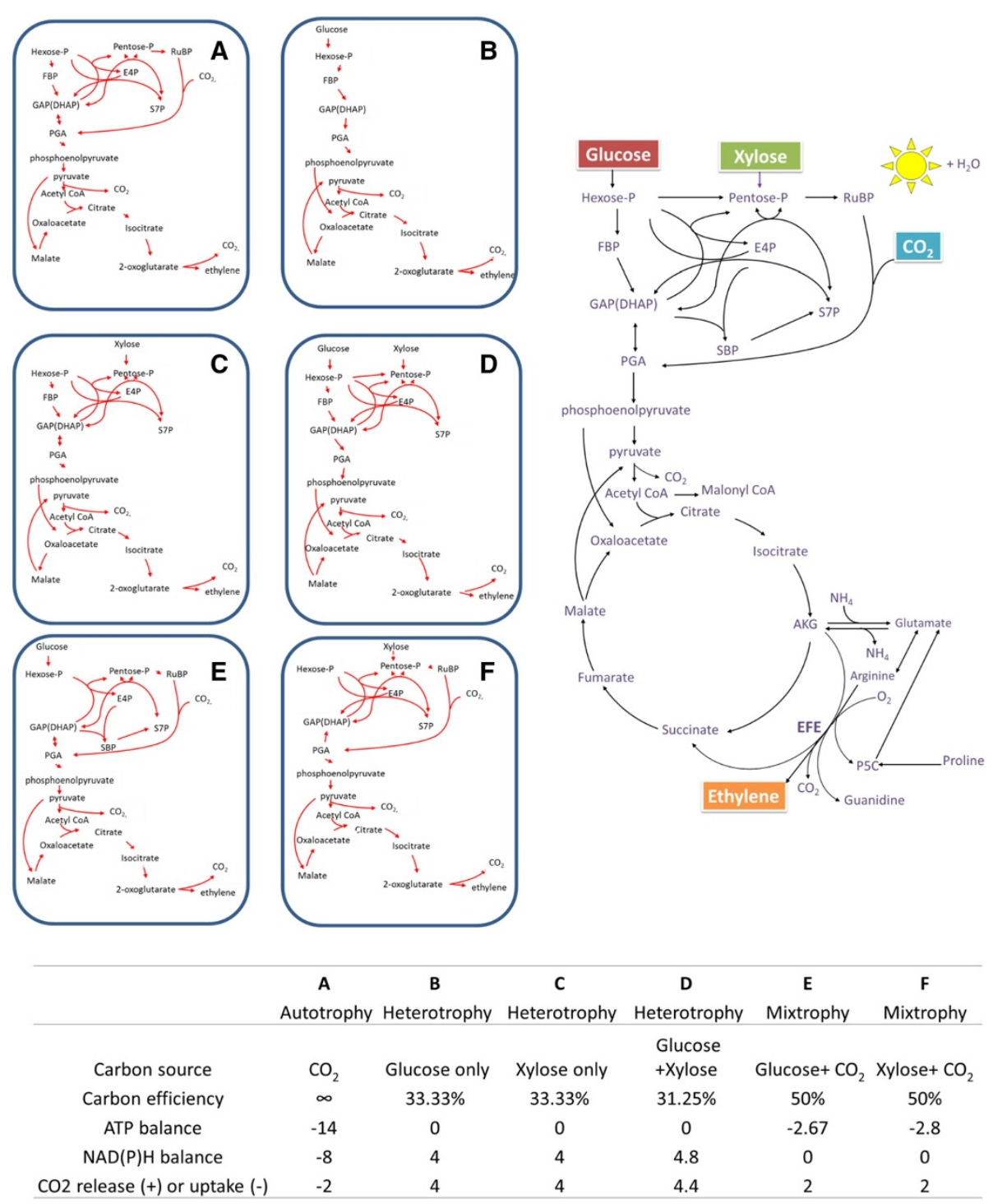

Figure 3 Various metabolic pathways towards ethylene production. De novo synthesis of ethylene by biological systems can be realized by using either organic or inorganic substrates (for example, glucose, xylose and $\mathrm{CO}_{2}$ ) in a global metabolic network (top right). Detailed in panels are the metabolic routes applying various combinations of substrates: (A) $\mathrm{CO}_{2}$ only (autotrophic), (B) Glucose only (heterotrophic), (C) xylose only (heterotrophic), (D) glucose + xylose (heterotrophic), (E) glucose $+\mathrm{CO}_{2}$ (mixotrophic), and (F) xylose $+\mathrm{CO}_{2}$ (mixotrophic). Corresponding carbon efficiency or yield (carbon stored in ethylene/carbon uptake), $\mathrm{CO}_{2}$ release/uptake, and cofactor balances in each panel are normalized to the formation of 1 mole of ethylene, and presented in the table (bottom right). Note that positive cofactor balances represent net production, while negative ATP or NADPH balances require cofactor supply from elsewhere (for example photosynthetic light reactions). The stoichiometries are calculated with computational analysis through determination of elementary modes for a given reaction system [34,35]. For computational analysis, all possible routes for conversion of organic/inorganic carbons to ethylene were considered. The reaction for ethylene production in panels (A-F) is defined as: a-ketoglutarate $=$ ethylene $+3 \mathrm{CO}_{2}$. Side reaction of ELE is not taken into account, because of the controversial and uncertain stoichiometry. $\mathrm{CO}_{2}$, carbon dioxide; DHAP, dihydroxyacetone phosphate; E4P, erythrose-4-phosphate; FBP, Fructose 1,6-bisphosphate; GAP, glyceraldehyde-3-phosphate; Hexose-P, hexose 6-phosphate; Pentose-P, pentose 5-phosphate; PGA, phosphoglycerate; RuBP, ribulose 1,5-bisphosphate; S7P, sedoheptulose-7phosphate; SBP, sedoheptulose 1,7-bisphosphate.

ethylene conversion from common substrates, including $\mathrm{CO}_{2}$ (via photosynthesis), glucose, and xylose, all possible feedstocks for the heterologous EFE-expressing hosts outlined in the next section. Figure 3A represents the classic photoautotrophic pathway for the conversion of $\mathrm{CO}_{2}$ to ethylene via the Calvin Benson Bassham cycle, and shows net carbon uptake and consumption of ATP and reductant (generated by photosynthetic light reactions). Figure 3B represents glycolysis from hexose to ethylene, resulting in $33.33 \%$ carbon yield and net reducing 
equivalents via the $\mathrm{NAD}(\mathrm{P})^{+}$-dependent activities of glyceraldehyde 3-phosphate dehydrogenase, pyruvate dehydrogenase, and isocitrate dehydrogenase (ICD). To reach the maximum theoretical yield, six carbons of a glucose molecule are split into two three-carbon units via glycolysis, one carbon is gained from the $\mathrm{C} 4$ route, and a total of five carbons are lost via pyruvate dehydrogenase (one carbon), ICD (one carbon), and EFE (three carbons), respectively. Utilization of xylose (Figure 3C) or mixed sugars (xylose plus glucose, Figure 3D) bypasses glycolytic flux via the pentosephosphate pathway, resulting in a similar ratio of reductant production and carbon yield as seen for glycolysis (Figure 3B). The routes outlined in Figure 3E and Figure 3F utilize the $\mathrm{CO}_{2}$-fixing enzyme ribulose1,5-bisphosphate carboxylase/oxygenase (Rubisco) as a non-oxidative shunt, reducing total carbon release in xylose-feeding or glucose-feeding systems, respectively. The additional one-pass flux through Rubisco increases carbon yield to $50 \%$, but there is a trade-off at the cost of increased cofactor requirements. Comparison of these metabolic routes reveals that photobiological conversion of $\mathrm{CO}_{2}$ to ethylene represents a carbon-negative process with a high demand for photosynthetically derived ATP and reducing equivalents, while heterotrophic ethylene production from hexose/pentose has no net cofactor input but still results in carbon loss. Therefore, strategies to recycle/avoid lost carbon must be considered. Recruiting both photosynthetic (could be more than one pass) and sugar-utilizing pathways in a mixotrophic mode should result in a higher theoretical yield and lower cofactor requirement simultaneously. In addition, carbon yield may be improved with pathways that bypass pyruvate dehydrogenase, or with a more efficient EFE (previous section).

Besides identifying the optimal pathways, metabolic bottlenecks and competing pathways can be revealed by systems biology approaches such as flux balance analysis (FBA) and metabolic flux analysis (MFA). MFA can help identify rate-limiting factors that control flux through $\mathrm{EFE}$, and can be used to measure EFE and competing fluxes in vivo. An analysis of metabolism towards ethylene formation was performed in genetically engineered S. cerevisiae using FBA [36], which used linear optimization to determine the steady-state reaction flux distribution in a mathematic network by maximizing ethylene production as an objective function [37]. In that study, either S-adenosylmethionine-dependent (via ACC) or AKG-dependent EFE were added into the reaction network, and optimized for maximal ethylene formation. The optimal ethylene yields calculated for the two systems were both in the range of 7-8 moles of ethylene/ 10 moles of glucose, or a carbon yield of 23.33 to $26.67 \%$ [36]; the maximal theoretical yield would be $33.33 \%$ when only minimal enzyme sets for ethylene production are considered (see above). Potential strategies to increase ethylene formation were also analyzed. The authors suggested that supplementation of exogenous proline, using a solely NAD-coupled glutamate dehydrogenase (catalyzes glutamate to AKG), and use of glutamate as the nitrogen source could increase ethylene formation. The study also indicated that computational results are close to experimentally observed values when additional constraints such as a constraint on respiratory capacity (for example, limiting $\mathrm{O}_{2}$ or not) are defined. Future work to identify the most efficient routes to ethylene production should include isotope labeling such as ${ }^{13} \mathrm{C}$ MFA [38,39] to allow for profiling of actual flux maps to complement the in silico modeling approaches.

\section{Heterologous expression of EFE and ethylene production}

Metabolic engineering to improve ethylene production and better understanding of metabolic flux to ethylene are necessary to realize bioethylene production on an industrial scale. As only one gene (efe) is necessary for ethylene production from common metabolites, it is of great interest to study the heterologous expression of EFE in organisms that can utilize a variety of feedstocks. Collectively, ethylene production has been successfully demonstrated in engineered microbes utilizing diverse renewable resources such as sunlight, cellulose, or biomass-derived glucose.

\section{E. coli and S. cerevisiae}

The earliest efforts at heterologous expression of EFE involved cloning of the Kudzu efe gene (with its native promoter) into a high-copy pUC19 vector and expressing it in E. coli, resulting in measurable ethylene production (Table 1) [15], and revealing that efe is sufficient for ethylene production in a foreign host. In a follow-up study, increased EFE expression from a lac promoter on a high-copy pUC18 vector, or from a tac promoter on a medium-copy pBR322 vector, led to much higher activities when cultures were grown at $25^{\circ} \mathrm{C}$ (Table 1, [16]). Alternatively, when cultures were grown at $37^{\circ} \mathrm{C}$, very low activities were detected at all stages, in agreement with the short half-life (3.3 minutes) of the EFE protein at $37^{\circ} \mathrm{C}$. Furthermore, raising the temperature from $25^{\circ} \mathrm{C}$ to $37^{\circ} \mathrm{C}$ led to increased localization of the protein in inclusion bodies. Interestingly, a short peptide (15 amino acids) from LacZ at the $\mathrm{N}$-terminus in the strain overexpressing EFE (from the lac promoter) led to decreased localization of EFE in inclusion bodies, but activity at $37^{\circ} \mathrm{C}$ remained low [16], suggesting that other factors probably also affect EFE activity and stability.

In addition to levels of active EFE, substrate availability may also limit ethylene production in heterologous expression systems. When Kudzu EFE was expressed under the constitutive $n p t$ promoter from a low-copy 
Table 1 Ethylene productivities in EFE-expressing microbes

\begin{tabular}{|c|c|c|c|c|c|c|}
\hline Host & $\begin{array}{l}\text { Native, vector, or } \\
\text { integrated EFE expression }\end{array}$ & Promoter & Temperature, ${ }^{\circ} \mathrm{C}$ & Feedstock & $\begin{array}{l}\text { Rate of ethylene } \\
\text { production. }(\mu \mathrm{mol} / \mathrm{gCDW} / \mathrm{h})\end{array}$ & Ref. \\
\hline Pseudomonas syringae (Kudzu) & Native & Kudzu & 30 & $\mathrm{LB}+0.5 \%$ glucose & 39.0 & 18 \\
\hline P. syringae (Kudzu) & Native + vector (RS1010) & Kudzu, npt & 30 & $\mathrm{LB}+0.5 \%$ glucose & 312.0 & 18 \\
\hline Escherichia coli (JM109) & Vector (pUC19) & Kudzu & 37 & Modified LB & 10.9 & 15 \\
\hline E. coli (DH5a) & Vector (pUC18) & lac & 25 & LB & 625.0 & 16 \\
\hline E. coli (JM109) & Vector (pBR322) & tac & 25 & LB & 412.9 & 16 \\
\hline E. coli (JM109) & Vector (RS1010) & npt & 30 & $L B+0.5 \%$ glucose & 55.2 & 18 \\
\hline E. coli (DH5a) & Vector (RS1010) & lac/trc & 30 & LB & 22.8 & 26 \\
\hline E. coli (MG155) & Vector (pBR322) & psbA & 30 & M9 $+1 \%$ glucose & 30.0 & UP \\
\hline Saccharomyces cerevisiae (batch) & Vector (pYX212) & tpi & 30 & YNB + $1 \%$ glucose + glutamate & 21.4 & 17 \\
\hline S. cerevisiae (chemostat) & Vector (pYX212) & tpi & 30 & $\mathrm{CBS}+1 \%$ glucose $+\left(\mathrm{NH}_{4}\right)_{2} \mathrm{SO}_{4}$ & 1083.8 & 42 \\
\hline S. cerevisiae (chemostat) & Vector (pYX212) & tpi & 30 & CBS $+1 \%$ glucose + glutamate & 1151.5 & 41 \\
\hline S. cerevisiae (chemostat) & Vector (pYX212) & tpi & 30 & CBS $+1 \%$ glucose + glutamate + arginine & 492.0 & 42 \\
\hline Synechococcus 7942 & Vector (pUC303) & psbA1 & 25 & BG11 & 84.8 & 23 \\
\hline Synechococcus 7942 & Integrated (psbAl) & psbA1 & 28 & BG11 & 80.5 & 24 \\
\hline Synechocystis 6803 & Vector (RS1010) & $\mathrm{lac} / \mathrm{trc}$ & 30 & BG11 & 26.0 & 26 \\
\hline Synechocystis 6803 & Integrated (slr0168) & psbA & 30 & BG11 & 111.6 & UP \\
\hline Trichoderma viride & Integrated (random) & cbhl & 30 & $\mathrm{MM}+2 \%$ cellulose $+0.2 \%$ peptone & 0.093 & 19 \\
\hline Trichoderma reesei & Integrated (random) & pgk & 30 & $M M+2 \%$ wheat straw & 0.716 & 20 \\
\hline Pseudomonas putida & Vector (RS1010) & npt & 30 & $\mathrm{LB}+0.5 \%$ glucose & 1050.0 & 18 \\
\hline P. putida & Integrated (five 165 rDNA sites) + vector (pBBR1MCS2) & rrn & 28 & LB & 2859.2 & 48 \\
\hline
\end{tabular}

For comparison, reported rates have been converted to $\mu \mathrm{mol} / \mathrm{gCDW} / \mathrm{h}$.

$\mathrm{CDW}$, cell dry weight; EFE, ethylene-forming enzyme. 
plasmid (RS1010) in E. coli, P. putida, and P. syringae (containing native gene + plasmid-based overexpression), $P$. putida exhibited the highest maximal rates of ethylene production (Table 1) [18]. Maximal activity in all three overexpression systems occurred early in growth and fell off rapidly, consistent with previous observations in $E$. coli [16]. Additionally, in vivo EFE activities (intracellular substrates only) of cultures sampled at time points with maximal production rates were compared with those in vitro (exogenously added substrates at saturating levels). These comparisons revealed that although WT P. syringae had similar EFE activities in vivo and in vitro, the in vitro activities from the E. coli, P. putida, and the $P$. syringae overexpressing strain, were, respectively 5fold, 20-fold, and 40-fold higher than those seen in vivo suggesting that substrate availability limits in vivo activity [18]. Zhang et al. recently reported that intracellular levels of AKG reached their highest levels in early growth [40], consistent with the above observation that AKG levels may be limiting.

Pirkov et al. observed that in S. cerevisiae, ethylene production nearly tripled when the nitrogen source in minimal media (1.0\% glucose) was changed from ammonium to glutamate in batch cultures when the Kudzu efe gene was expressed by a strong, constitutive tpi promoter on a multicopy $2 \mu$ plasmid (Table 1) [17], in agreement with an in silico production model (see previous section) [36]. This model also revealed that experimentally measured ethylene yields were consistent with the yields predicted under limited respiration (Table 1) [36], suggesting that $\mathrm{O}_{2}$ availability is necessary for maximal ethylene production. In a more recent study, ethylene production was further analyzed in a chemostat with increased $\mathrm{O}_{2}$, leading to improvement in ethylene production by more than 53 -fold over that seen in batch cultures (Table 1) [41]. When the nitrogen source was changed from ammonium to glutamate, growth was improved, but no change in specific ethylene productivity was seen, suggesting that the improvement observed with glutamate addition to batch cultures was due to cell growth and not increased EFE productivity (Table 1) [41]. Furthermore, addition of the EFE substrate arginine actually reduced ethylene productivity by over half (Table 1) [41]. The authors postulated that addition of arginine may result in a "push" towards the succinateforming sub-reaction proposed by the dual-circuit mechanism [41]. Together, these studies highlight that beyond strategies to improve EFE stability, further analysis of substrate enrichment and increased $\mathrm{O}_{2}$ availability are necessary to maximize ethylene production.

\section{Cyanobacteria}

To link ethylene production to photosynthetic $\mathrm{CO}_{2}$ fixation, the efe gene from the Kudzu strain was heterologously expressed in cyanobacteria in a number of studies. In Synechococcus elongatus sp. PCC 7942, vectorbased expression of EFE was first explored from a lowcopy pUC303 vector [22,23]. Interestingly, unlike that seen for heterologous expression in E. coli, the in vivo and in vitro activities were comparable, suggesting that substrates for the EFE reaction are not limiting in Synechococcus [23]. When plasmid-based expression of EFE was analyzed using a variety of promoters, a native $p s b A 1$ promoter exhibited the highest activity (Table 1) [23], although vectors containing more than 100-bp homology to this native $p s b A 1$ promoter region were unstable. Plasmid instability was correlated with slow growth, bleaching, and a decreased $\mathrm{CO}_{2}$ to ethylene partition rate compared with strains containing vectors with no/low native sequence (100 bp or fewer), and lower rates of ethylene production [23]. The authors postulated that decreased fitness could be a result of plasmid loss (loss of antibiotic resistance in the presence of antibiotic) and/or metabolic stress linked to EFE activity, as the addition of ethylene to cultures did not affect growth [23].

To address such instability issues, the efe gene was integrated at the psbA1 locus in S. elongatus sp. PCC $7942[24,42,43]$. When a kanamycin resistance gene was additionally integrated behind the efe gene, ethylene production was stable over 30 generations [42,43]. A markerless insertion of efe at the same locus exhibited rates of ethylene production that were four times higher than in strains containing the integrated kanamycin resistance gene [24], exhibiting rates similar to those in the best plasmid-based expression strains (Table 1) [23,24]. These markerless integration strains similarly exhibited defective growth and metabolic stress when active EFE was expressed $[23,24]$. The authors suggested that with increased ethylene production, levels of AKG become limiting, hence shifting glutamate to AKG instead of to bilin production [44], leading to cell bleaching and slowed growth rates. It is unknown whether increased availability of AKG (as well as arginine) would increase ethylene production and/or rescue the growth defects in these strains.

Expression of Kudzu EFE from the low-copy RSF1010 plasmid was compared in E. coli and the unicellular cyanobacterium Synechocystis sp. PCC6803 [26]. Unlike plasmid-based expression in Synechococcus, expression of EFE from this vector did not lead to plasmid instability. The highest rates of production were achieved for both E. coli and Synechocystis when the trc or lacO-1 promoters were utilized, although expression in Synechocystis was independent of IPTG addition (Table 1 [26]).

The efe gene from the Kudzu strain has also been codon-optimized and integrated into the genome of Synechocystis [25]. Stable expression of the EFE was achieved and optimized [25] using a constitutive, highlevel pea plant chloroplast $p s b A$ promoter $\left(\sigma^{70}\right.$ 
consensus [45]) to drive its expression when integrated at the slr0168 neutral-site locus [46]. Current work to increase EFE expression levels has led to even higher rates of ethylene production (Table 1) (Jianping Yu, unpublished). It is currently unknown whether the EFE sequestration or stability issues discussed above similarly affect ethylene production in this cyanobacterium.

\section{Cellulolytic fungi and microbes that utilize diverse feedstocks}

With many fungi exhibiting strong cellulolytic activity, expression of EFE in fungal hosts provides a promising route for ethylene production from renewable biomass. Tao et al. reported the successful heterologous expression of an integrated $P$. syringae pv. glycinea efe gene driven by the strong cbhI promoter in T. viride [19]. Maximal production rates were observed when $2.0 \%$ cellulose and $0.2 \%$ peptone were used as carbon sources, with the addition of peptone having the most significant impact on production (Table 1) [19]. Another cellulolytic fungus, $T$. reese $i$, was also analyzed as a host for expression of efe from P. syringae pv. glycinea [20]. The efe gene was randomly integrated into the genome and expressed by a variety of promoters, and resultant strains were screened for the highest rates of production. A strain expressing efe from the $p g k$ promoter of $T$. reese $i$ demonstrated the highest activity (Table 1) [20].

P. putida is a Gram-negative soil bacterium with a diverse metabolism that has potential for the production of a variety of compounds using various waste streams as feedstock. Based on the high ethylene production rates exhibited by $P$. putida expressing Kudzu EFE from a plasmid (see above), Wang et al. designed a vector to integrate multiple copies of the efe gene (from P. syringae pv. glycinea) into the $16 \mathrm{~S}$ rDNA sites of $P$. putida [47]. The use of this construct led to the integration of 3-5 copies of the efe gene, with expression driven by the native $r r n$ promoter. Ethylene production rates increased with increasing copy number, with the highest rate achieved in the strain containing five integrated copies in addition to expression from a medium-copy, broadhost range plasmid. This strategy ultimately increased ethylene production and glucose-to-ethylene conversion to the highest rates by native and recombinant organisms reported to date (Table 1) [47].

\section{Harvesting of biologically produced ethylene}

One important consideration for the biological production of ethylene is harvesting. In the petrochemical industry, ethylene is typically harvested from a gaseous mixture via cryogenic distillation [48], which is energyintensive but is capable of harvesting multiple gaseous products in the mixture. Other methods include solvent extraction, pressure swing adsorption using zeolites, and membrane separation. Special consideration must be implemented in the harvesting of biologically produced ethylene, depending on the gas composition in the mixture. It is expected that besides ethylene, there may also be $\mathrm{CO}_{2}$, water vapor, $\mathrm{N}_{2}$, and $\mathrm{O}_{2}$ present in the biologically derived gaseous stream. When $\mathrm{O}_{2}$ is co-produced with ethylene in a photosynthetic system, there is an important safety issue regarding the flammability of ethylene in the presence of $\mathrm{O}_{2}(2.7$ to $36 \% \mathrm{v} / \mathrm{v}$ [49]). Therefore, engineering designs must be included to mitigate this risk. Biologically produced ethylene is expected to be free of metals and other contaminants commonly found in fossil-derived ethylene stream, and therefore may become a preferred feedstock for highpurity chemicals and clean fuels.

\section{Future research directions}

The development of bioethylene technologies is in its infancy. In order to confer a major impact in displacing fossil-derived feedstocks, advances in many research areas are needed to improve ethylene production strains, cultivation systems, and harvesting technologies. Work is ongoing to conduct a technoeconomic analysis of bioethylene production in a cyanobacterial system. Its outcomes will provide parameters to guide future directions in research and development.

As discussed above, fundamental knowledge of the structure, function, and reaction mechanism of EFE is currently lacking. The analysis of EFE and its related sequences and structures to identify conserved regions and a putative enzyme active site will aid research to evaluate these features and the proposed dual-circuit catalytic mechanism. A crystal structure of EFE will additionally enhance our understanding of this enzyme and guide protein engineering towards increased carbon yield and thermal stability. Furthermore, accurate reaction stoichiometry coupled with carbon flux analysis will guide metabolic pathway engineering to construct more efficient production route(s).

The advent of synthetic biology will also accelerate strain development by optimizing the design of pathways for high-yield ethylene production. To realize the full potential of a synthetic biology-based engineering approach, high-throughput screening/selection tools are needed to monitor levels of ethylene and its precursors. Currently, genetically encoded, sensor-based screens have been developed for AKG [40] and arginine [50], and a direct ethylene sensor could potentially be constructed based on the ethylene receptor in plants and cyanobacteria [51].

Lastly, for scaled-up production, inexpensive bioreactors must be developed with enhanced $\mathrm{O}_{2}$ mass transfer for non-photosynthetic systems, light delivery for photosynthetic systems, and associated harvesting systems tailored to a biologically derived gas stream. 


\section{Conclusions}

During the course of evolution, microbes have developed multiple ethylene-producing pathways to take advantage of ethylene-responsive mechanisms in plants and facilitate the successful invasion of plant tissue. The outcome may be beneficial only to the microbes in the case of pathogenesis, or it may be mutually beneficial in the possible case of symbiosis. Nevertheless, the underlying mechanism governing EFE catalysis remains as an emerging research topic for the production of bioethylene. With the advent of synthetic biology tools and advanced analytical capabilities, robust ethylene production via EFE can be exploited in heterologous systems for production of this versatile feedstock from diverse renewable resources such as biomass, sunlight, and $\mathrm{CO}_{2}$. The successful outcome will reduce our dependence on fossil fuels, and provide a viable feedstock for bio-based chemicals and fuels.

\section{Abbreviations}

2OG: 2-oxoglutarate; AKG: alpha-ketoglutarate; ACC: 1-aminocyclopropane-1carboxylic acid; CBB cycle: Calvin Benson Bassham cycle; EFE: ethyleneforming enzyme; FBA: flux balance analysis; KMBA: 2-keto-4-methylthiobutyric acid; MFA: metabolic flux analysis; P5C: L-delta-1-pyrroline-5-carboxylate; Rubisco: ribulose-1,5-bisphosphate carboxylase/oxygenase; SAM: S-adenosylmethionine

\section{Competing interests}

The authors declare they have no competing interest in this work.

\section{Authors' contributions}

CE, RG, PCM and JY conceived the project and edited the manuscript. All authors analyzed and interpreted data and drafted various parts. In particular, PCM drafted the introduction; JY drafted "EFE discovery and future research directions"; WXu drafted "EFE diversity and features"; JU and JY drafted "EFE reaction mechanism; WXiong drafted "EFE and metabolic modes"; CE and SL drafted "EFE heterologous expression and ethylene production"; LT drafted "ethylene harvesting"; RG drafted the conclusion. All authors read and approved the final manuscript.

\section{Acknowledgements}

This study was supported by the US Department of Energy Office of Science Biological and Environmental Research (to CE, SL, PCM, JY, and RG); the Office of Energy Efficiency and Renewable Energy Bioenergy Technologies Office (LT, JU, and JY); and a National Renewable Energy Laboratory Director's Postdoctoral Fellowship (WXiong).

\section{Author details \\ ${ }^{1}$ Biosciences Center, National Renewable Energy Laboratory, 15013 Denver West Parkway, Golden, CO 80401, USA. ²Department of Chemistry, University of Louisiana at Lafayette, Lafayette, LA 70503, USA. ${ }^{3}$ Renewable and Sustainable Energy Institute, University of Colorado Boulder, Boulder, CO 80309, USA.}

Received: 19 December 2013 Accepted: 13 February 2014 Published: 3 March 2014

\section{References}

1. Consulting SRI: CEH Marketing Research Report: Ethylene. Englewood, Colorado, USA: SRI International; 2011

2. Ipatieff VN, Corson BB: Gasoline from ethylene by catalytic polymerization. Ind Eng Chem 1936, 28:860-863.

3. Kusmiyati A, NAS: Production of gasoline range hydrocarbons from catalytic reaction of methane in the presence of ethylene over W/HZSM-5. Catal Today 2005, 106:271-274.
4. Worrell E, Phylipsen D, Einstein D, Martin N: Energy use and energy intensity of the US chemical industry. Berkeley California, USA: Lawrence Berkeley National Laboratory; 2000.

5. Johnson PR, Ecker JR: The ethylene gas signal transduction pathway: a molecular perspective. Annu Rev Genet 1998, 32:227-254.

6. Wang $\mathrm{KL}$, Li H, Ecker JR: Ethylene biosynthesis and signaling networks. Plant Cell 2002, 14(Suppl):S131-151.

7. Weingart $H$, Ullrich $H$, Geider $K$, Volksch B: The role of ethylene production in virulence of Pseudomonas syringae pvs. glycinea and phaseolicola. Phytopathology 2001, 91:511-518.

8. Mansouri S, Bunch AW: Bacterial ethylene synthesis from 2-oxo-4-thiobutyric acid and from methionine. J Gen Microbiol 1989, 135:2819-2827.

9. Shipston N, Bunch AW: The physiology of L-methionine catabolism to the secondary metabolite ethylene by Escherichia coli. J Gen Microbiol 1989, 135:1489-1497.

10. Biale JB: Effect of emanations from several species of fungi on respiration and color development of citrus fruits. Science 1940, 91:458-459.

11. Winston JR, Miller EV, Fisher DF: Production of epinasty by emanations from normal and decaying citrus fruits and from Penicillium digitatum. J Agric Res 1940, 60:269-277.

12. Young RE, Pratt HK, Biale JB: Identification of ethylene as a volatile product of the fungus Penicillium digitatum. Plant Physio/ 1951, 26:304-310.

13. Jacobsen DW, Wang $\mathrm{CH}$ : The biogenesis of ethylene in Penicillium digitatum. Plant Physiol 1959-1966, 1968:43.

14. Nagahama K, Ogawa T, Fujii T, Tazaki M, Tanase S, Morino Y, Fukuda H: Purification and properties of an ethylene-forming enzyme from Pseudomonas syringae Pv phaseolicola Pk2. J Gen Microbiol 1991, 137:2281-2286.

15. Fukuda H, Ogawa T, Ishihara K, Fujii T, Nagahama K, Omata T, Inoue Y, Tanase S, Morino Y: Molecular cloning in Escherichia coli, expression, and nucleotide sequence of the gene for the ethylene-forming enzyme of Pseudomonas syringae Pv phaseolicola Pk2. Biochem Bioph Res Co 1992, 188:826-832.

16. Ishihara $K$, Matsuoka M, Inoue $Y$, Tanase $S$, Ogawa T, Fukuda H: Overexpression and in vitro reconstitution of the ethylene-forming enzyme from Pseudomonas syringae. J Ferment Bioeng 1995, 79:205-211.

17. Pirkov I, Albers E, Norbeck J, Larsson C: Ethylene production by metabolic engineering of the yeast Saccharomyces cerevisiae. Metab Eng 2008, 10:276-280

18. Ishihara K, Matsuoka M, Ogawa T, Fukuda H: Ethylene production using a broad-host-range plasmid in Pseudomonas syringae and Pseudomonas putida. J Ferment Bioeng 1996, 82:509-511.

19. Tao L, Dong HJ, Chen X, Chen SF, Wang TH: Expression of ethylene-forming enzyme (EFE) of Pseudomonas syringae pv. glycinea in Trichoderma viride. Appl Microbiol Biot 2008, 80:573-578.

20. Chen X, Liang Y, Hua J, Tao L, Qin W, Chen S: Overexpression of bacterial ethylene-forming enzyme gene in Trichoderma reesei enhanced the production of ethylene. Int J Biol Sci 2010, 6:96-106.

21. Araki S, Matsuoka M, Tanaka M, Ogawa T: Ethylene formation and phenotypic analysis of transgenic tobacco plants expressing a bacterial ethylene-forming enzyme. Plant \& cell physiology 2000, 41:327-334.

22. Fukuda H, Sakai M, Nagahama K, Fujii T, Matsuoka M, Inoue Y, Ogawa T: Heterologous expression of the gene for the ethylene-forming enzyme from Pseudomonas syringae in the cyanobacterium Synechococcus. Biotechnol Lett 1994, 16:1-6.

23. Sakai M, Ogawa T, Matsuoka M, Fukuda H: Photosynthetic conversion of carbon dioxide to ethylene by the recombinant cyanobacterium, Synechococcus sp. PCC 7942, which harbors a gene for the ethylene-forming enzyme of Pseudomonas syringae. J Ferment Bioeng 1997, 84:434-443.

24. Takahama K, Matsuoka M, Nagahama K, Ogawa T: Construction and analysis of a recombinant cyanobacterium expressing a chromosomally inserted gene for an ethylene-forming enzyme at the psbAl locus. J Biosci Bioeng 2003, 95:302-305.

25. Ungerer J, Tao L, Davis M, Ghirardi M, Maness PC, Yu JP: Sustained photosynthetic conversion of $\mathrm{CO}_{2}$ to ethylene in recombinant cyanobacterium Synechocystis 6803. Energ Environ Sci 2012, 5:8998-9006.

26. Guerrero F, Carbonell V, Cossu M, Correddu D, Jones PR: Ethylene synthesis and regulated expression of recombinant protein in Synechocystis sp PCC 6803. PLOS One 2012, 7:e50470. doi:10.1371/ journal.pone.0050470.

27. Fukuda H, Fujii T, Ogawa T: Preparation of a cell-free ethylene-forming system from Penicillium digitatum. Agr Biol Chem Tokyo 1986, 50:977981 
28. Fukuda H, Kitajima H, Fujii T, Tazaki M, Ogawa T: Purification and some properties of a novel ethylene-forming enzyme produced by Penicillium digitatum. FEMS Microbiol Lett 1989, 59:1-5.

29. Freebairn HT, Buddenhagen IW: Ethylene production by Pseudomonas solanacearum. Nature 1964, 202:313-314.

30. Hausinger RP: Fell/alpha-ketoglutarate-dependent hydroxylases and related enzymes. Crit Rev Biochem Mol Biol 2004, 39:21-68.

31. Zhang Z, Ren JS, Clifton IJ, Schofield CJ: Crystal structure and mechanistic implications of 1-aminocyclopropane-1-carboxylic acid oxidase-the ethylene-forming enzyme. Chemistry and Biology 2004, 11:1383-1394.

32. Nagahama K, Yoshino K, Matsuoka M, Tanase S, Ogawa T, Fukuda H: Site-directed mutagenesis of histidine residues in the ethylene-forming enzyme from Pseudomonas syringae. J Ferment Bioeng 1998, 85:255-258.

33. Fukuda H, Ogawa T, Tazaki M, Nagahama K, Fujii T, Tanase S, Morino Y: Two reactions are simultaneously catalyzed by a single enzyme: the argininedependent simultaneous formation of two products, ethylene and succinate, from 2-oxoglutarate by an enzyme from Pseudomonas syringae. Biochem Bioph Res Co 1992, 188:483-489.

34. Schuster S, Dandekar T, Fell DA: Detection of elementary flux modes in biochemical networks: a promising tool for pathway analysis and metabolic engineering. Trends Biotechnol 1999, 17:53-60.

35. Rodriguez Fl, Esch JJ, Hall AE, Binder BM, Schaller GE, Bleecker AB: A copper cofactor for the ethylene receptor ETR1 from Arabidopsis. Science 1999, 283:996-998.

36. Schuster S, Fell DA, Dandekar T: A general definition of metabolic pathways useful for systematic organization and analysis of complex metabolic networks. Nat Biotechnol 2000, 18:326-332.

37. Larsson C, Snoep JL, Norbeck J, Albers E: Flux balance analysis for ethylene formation in genetically engineered Saccharomyces cerevisiae. IET Syst Biol 2011, 5:245-251.

38. Raman K, Chandra N: Flux balance analysis of biological systems: applications and challenges. Brief Bioinform 2009, 10:435-449.

39. Wiechert W: 13C metabolic flux analysis. Metab Eng 2001, 3:195-206.

40. Wiechert W, Mollney M, Petersen S, de Graaf AA: A universal framework for 13C metabolic flux analysis. Metab Eng 2001, 3:265-283.

41. Zhang C, Wei ZH, Ye BC: Quantitative monitoring of 2-oxoglutarate in Escherichia coli cells by a fluorescence resonance energy transfer-based biosensor. Appl Microbiol Biot 2013, 97:8307-8316.

42. Johansson N, Quehl P, Norbeck J, Larsson C: Identification of factors for improved ethylene production via the ethylene forming enzyme in chemostat cultures of Saccharomyces cerevisiae. Microb Cell Fact 2013, 12:89.

43. Wang JS, Araki T, Matsuoka M, Ogawa T: A model of photoinhibition related to mRNA instability in ethylene production by a recombinant cyanobacterium. J Theor Biol 2000, 202:205-211.

44. Wang JS, Araki T, Ogawa T, Sakai M, Matsuoka M, Fukuda H: Prediction of photosynthetic production rate of ethylene using a recombinant cyanobacterium. J Theor Biol 1999, 196:9-17.

45. Grimm B, Bull A, Breu V: Structural genes of glutamate 1-semialdehyde aminotransferase for porphyrin synthesis in a cyanobacterium and Escherichia coli. Mol Gen Genet 1991, 225:1-10.

46. Brixey PJ, Guda C, Daniell H: The chloroplast psbA promoter is more efficient in Escherichia coli than the T7 promoter for hyperexpression of a foreign protein. Biotechnol Lett 1997, 19:395-399.

47. Kommalapati M, Hwang HJ, Wang HL, Burnap RL: Engineered ectopic expression of the psbA gene encoding the photosystem II D1 protein in Synechocystis sp. PCC6803. Photosynth Res 2007, 92:315-325.

48. Wang JP, Wu LX, Xu F, LV J, Jin HJ, Chen SF: Metabolic engineering for ethylene production by inserting the ethylene-forming enzyme gene (efe) at the 16S rDNA sites of Pseudomonas putida KT2440. Bioresour Technol 2010, 101:6404-6409.

49. Zimmermann H, Walzl R: Ethylene. Hoboken, New Jersey, USA: In UIImann's Encyclopedia of Industrial Chemistry. Wiley-VCH Verlag GmbH \& Co. KGaA 2000. doi:10.1002/14356007.a10_045.pub3.
50. Zabetakis MG: Flammability characteristics of combustible gases and vapors. Bureau of Mines 1965, 627:50-51.

51. Schendzielorz G, Dippong M, Grunberger A, Kohlheyer D, Yoshida A, Binder S, Nishiyama C, Nishiyama M, Bott M, Eggeling L: Taking control over control: use of product sensing in single cells to remove flux control at key enzymes in biosynthesis pathways. ACS Synth Bio/ 2013, 3:21-29.

doi:10.1186/1754-6834-7-33

Cite this article as: Eckert et al:: Ethylene-forming enzyme and bioethylene production. Biotechnology for Biofuels 2014 7:33.

\section{Submit your next manuscript to BioMed Central and take full advantage of:}

- Convenient online submission

- Thorough peer review

- No space constraints or color figure charges

- Immediate publication on acceptance

- Inclusion in PubMed, CAS, Scopus and Google Scholar

- Research which is freely available for redistribution

Submit your manuscript at www.biomedcentral.com/submit
C BioMed Central 